\title{
The putative role of brain lymphatic system in ménière disease pathogenesis
}

\begin{abstract}
Ménière's disease (MD) is a chronic illness of the inner ear. It is characterised by intermittent episodes of vertigo lasting from minutes to hours, with sensor neural, usually fluctuating, hearing loss, tinnitus, and aural pressure. Diagnosis is mainly based on clinical course and MD is usually considered as a multifactorial disease. Thus there are not yet evidences of a specific treatment. Authors start from a particular case of MD patient that was completely healed after tonsillectomy, an absolute unusual treatment for this kind of disease. On the basis of Literature and their studies about the role of vascular, viral, allergologic and immunologic disorders in MD patients, Authors speculates about the Brain Lymphatic System as an unitary explanation of the effects of infective foci, like chronic palatine tonsils, viral infections, inner ear epitopes activation and allegro-immunologic disturbances in MD pathogenesis. This kind of approach, in the aim of the Authors, may help to better planning a tailored treatment for the patient.
\end{abstract}

Keywords: mèniére disease, tonsillectomy, allergy, viral infections, brain lymphatic system

\author{
Special Issue - 2018
}

\author{
Alpini Dario Carlo,, ${ }^{12}$ Di Berardino Federica, ${ }^{3}$ \\ Mattei Valentina,' De Munari Manuela, ${ }^{2}$ \\ Berardi Carlo' \\ 'IRCCS S Maria Nascente don Carlo Gnocchi Foundation, \\ Europe \\ ${ }^{2}$ Vertigo School, Europe \\ ${ }^{3}$ Department of Clinical Sciences and Community, University of \\ Milan, Europe
}

Correspondence: Alpini Dario Carlo,Vertigo School, via Lomellina 58, 20133 Milan, Europe,

Email vertigoschool5@gmail.com

Received: February 25, 2017 | Published: November 16, 2018

\section{Introduction}

Ménière's disease (MD) is a chronic illness of the inner ear with an incidence, in Europe about 50-200/100.000 a year. ${ }^{1}$ It is characterised by intermittent episodes of vertigo lasting from minutes to hours, with sensor neural, usually fluctuating, hearing loss, tinnitus, and aural pressure. Diagnosis is mainly based on clinical course and MD is usually considered as a multifactorial disease, thus there are not yet evidences for a specific treatment. ${ }^{2,3}$ The inner ear comprehends hearing and balance sensory organs it is deeply embedded into the petro us part of the temporal bone, its blood supply is provided through the labyrinthine artery, a branch of the anterior inferior cerebellar artery ( $>85 \%$ cases) or basilar artery ( $<15 \%$ cases), its drainage is formed by the labyrinthine veins that flow, via the inferior petro us sinus, into the Internal Jugular Veins (IJVs). ${ }^{4}$ The luminal compartment of the inner ear, known as the endolymphatic space, is separated by the abluminal compartment, the perilymphatic space, through highly specialized epithelial cells with tight junctions. The fluid volume within the bony labyrinth remains constant. Changes in the volumes of the endolymphatic and perilymphatic compartments are responses to osmotic gradients between the compartments themselves. ${ }^{5}$ MD is characterized by impairment of this balance between the compartments causing the so called Endolymphatic Hydrops (EH), usually considered the pathogenesis of MD. The EH associated with Ménière's disease has been correlated to many etiological factors able to alter the endolymphatic homeostasis, such as abnormalities in end lymph production or absorption, ${ }^{6}$ cervico-cephalic venous drainage impairment ${ }^{7-9}$ genetic anomalies, allergies, ${ }^{10}$ viral infections ${ }^{11}$ and autoimmunity or inflammatory processes..$^{12}$ It has been suggested that certain cases of MD may have an altered immunological background, which may be attributable to an autoimmune mechanism that depends on humoral and/or cellular responses leading to an altered bloodlabyrinth barrier. ${ }^{13}$ The underlying mechanism of EH is still debated, with some researches in favour of a purely hydraulic mechanism and others hypothesizing a control mechanism of ionic balance. Hydraulic hypothesis supports ES surgical treatment while Control hypothesis a pharmachologic and dietary approach.

\section{An intriguing case report}

We observed a particularly successfully complete resolution of MD in a young woman after tonsillectomy, an absolute atypical approach to this kind of disorder. Marta $\mathrm{C}$ was affected with an absolute right hearing loss occurred during infancy probably caused by a "viral" infection. When she was 21 Marta begun to manifest typical Ménière attacks with involvement of the left, healthy, ear. This condition is also called Delayed Hydrops, which is a MD that occurs many years after a sudden hearing loss. ${ }^{14}$ Generally speaking, involvement of the healthy ear is probably due to a cochlear autoimmune response activated by the initial viral infection. ${ }^{15}$ In the same period she began to alternate typical MD spells and "typical" acute tonsillitis. Antibiotics were effective during tonsillitis but they did not prove to be effective to prevent the acute episodes. Throat specimen during acute episodes and between them was always negative for a bacterial origin of chronic tonsillitis. On the other hand, an enzyme-linked immunosorbent assay (ELISA) was used to detect antibodies (IgM and IgG) to Herpes simplex type 1-2 (HSV-1, HSV-2), cytomegalovirus (CMV), varicella-zoster virus (VZV) and Ebstein-Barr (EBV). The presence of high level of CMV IgG was detected. On the basis of clinical and laboratory results the patient underwent tonsillectomy. Ménière attacks immediately stopped and after more than 10 years follow-up no more vertigo attacks occur and left, health, hearing is completely normal. Evidently, tonsillectomy removed infective foci able to activate a counter-inner ear response. However, the question is which is the putative link between palatine tonsils infections and Ménière Disease?

\section{Some further considerations about MD and immune system}

A similar question regards the role of virus and the role of allergy in MD pathogenesis A viral cause of MD has been sustained by some Authors. Gacek, for instance ${ }^{16,17}$ hypnotized a vestibular ganglionitis or a vestibular neuropathy induced by Herpes infections or re-activations . In our experience only in one case we could sustain a direct action 
of virus in MD when we had been able to measure anti-VZV IgM and IgG and Anti Heath Shock Protein (HSP)-70 antibodies in a patient in which both the early phase of VZV manifestation and a typical MD acute attack occurred at the same time. ${ }^{18}$ If a viral direct action on inner ear lacks of laboratory support, the role of allergy in MD seems more evident and sustainable. Derebery and Berliner describe three theories related to allergy in MD that focus on inflammation within the endolymphatic sac. ${ }^{19}$ First, the endolymphatic sac contains a fenestrated blood supply that may allow antigen entry leading to mast cell degranulation and inflammation. A second proposed mechanism involves circulating immune complexes that enter endolymphatic sac circulation and the stria vascularis leading to inflammation and increased permeability as well as fluid balance disruption. A final theory pertains to a viral antigen-allergic interaction. Viruses have been shown to exacerbate allergic symptoms by enhancing histamine release and can damage epithelial surfaces as well as trigger T-cell migration to the endolymphatic sac. ${ }^{20}$

\section{The question remains the same}

\section{How viruses may be effectively removed by the inner ear?}

Both inhalant and food allergies have been associated with Di Beradino et al. ${ }^{21}$ verified the incidence of gliadin IgE hypersensitivity ${ }^{21}$ and the effectiveness of gluten-free diet in MD patients. ${ }^{22}$ To sum up, also in the case of allergy a unitary explanation of damage and repair mechanisms seems to lack.

\section{The putative role of brain lymphatic system \\ Brain tissues are composed of three compartments}

Parenchyma (neural and glial cells), vascular system and then interstitial system (ISS). The brain ISS is an irregular, tortuous and narrow space among neural cells and capillaries and contains interstitial fluid (ISF) and extracellular matrix (ECM). ${ }^{23}$ Cerebro Spinal Fluid (CSF) and ISF are strictly functionally connected. It has been suggested that Cerebro Spinal Fluid (CSF) formation occurs by filtration and flux of fluid through the capillary walls, and that the respective volumes of CSF and interstitial fluid mainly depend on hydrostatic and osmotic forces between the CSF and brain parenchyma created by gradients of proteins and inorganic ions across the capillary membrane. CSF and ISF are continuously interchanging and the volume occupied by each compartment depends on hydrostatic and osmotic forces. This exchange is facilitated by convective influx of CSF along the periarterial space. From the subarachnoid space, CSF is driven into the Virchow-Robin spaces by a combination of arterial pulsatility, respiration, slow vasomotion, and CSF pressure gradients. The loose fibrous matrix of the perivascular space can be viewed as a low resistance highway for CSF influx. The cranial cavity is a space of fixed volume in which fluids are functionally connected: variations in intracranial blood volume produce fluctuations of intracranial pressure and, consequently, exchange of CSF between the intracranial and spinal subarachnoid spaces.

Brain ISF is a water solvent that contains ions, gaseous molecules and organic molecules bathing and surrounding the neural cells. ICF may be considered another pathway, besides arterial blood flow, to provide the substances that brain cells need for their metabolism. Furthermore, ISF is one of the most important drainage pathways of the clearance waste cerebral products of brain metabolism, including the inner ear, on top of the venous system. The waste clearance ISF based system has been called lymphatic System (GS) ${ }^{24}$ GS is a macroscopic waste clearance system that uses the perivascular tunnels, formed by astroglial cells, to promote efficient removal of soluble proteins and metabolites from the central nervous system. Three potentials ways have been suggested ${ }^{23}$ one runs along the wall of the ventricle through epyndemal cells, anther exists at the surface of the brain and spinal cord via pia-glial membranes; these two pathways involve direct exchanges between ISF and CSF the third pathway is the blood vessel wall, where ISF flows within the basement membrane directly opposite to blood flow and eventually reaching the deep cervical lymph nodes through the olfactory bulb and nasal lymphatics. Very recently ${ }^{25}$ a GS disorder h6s been specifically considered as potential mechanism underling open-angle glaucoma. In this eye disorder, even if path physiology remains uncertain, the lamina cribrosa seems to be the primary site of injury. Lamina cribrosa is a route by which GS promotes elimination of interstitial solutes, including beta-amyloid, along paravascular channels from the brain to the nose. Thus, it has been hypnotized that primary open-angle glaucoma may result from the restriction of normal Glymphatic flow at the level of lamina cribrosa. The role of a GS disturbance has been taken in account also for Ménière Disease pathogenesis ${ }^{26}$ because the cochlear aqueduct and the internal auditory canal communicate with the subarachnoid space from where CSF is driven into the Virchow-Robin spaces. GS works mainly during sleep and it is largely disengaged during wakefulness. ${ }^{27}$ Nakayama et al..$^{28}$ found in 35 patients that total sleeping time was significantly higher than in controls. Nevertheless, they also showed in these patients, for the first time, a poor quality of sleep with decreased deep sleep and an elevated arousal index allowing speculating an impairment of GS at least in some patients affected with MD. However, as far as the present case report regards, in our opinion, more than GS, is the Brain Lymphatic System (BLS) that has to be taken in account to understand as un unique phenomenon direct or undirected, through the activation of inner ear epitopes, ${ }^{29}$ the action of virus, allergic and immune reaction, and the resulting preventive actions. A true lymphatic vascular system draining brain interstitial fluids and macromolecules has been very recently described. ${ }^{30}$ Generally speaking, BLS is formed by a network of lymphatic vessels in the dura mater of the Central Nervous System (CNS) that drains out via the foramina of the skull base itself alongside arteries, specifically the major branches of the middle and anterior meningeal arteries and the pterygopalatine artery, cranial nerves, specifically I, V, IX, X and XIth, and veins. Lymphatic vessels have been observed in the dural lining of the cribriform plate where some vessels pass into the nose. ${ }^{31}$

Dura mater ${ }^{32,33}$ lymphatic vessels drains brain ISF into deep cervical lymph-nodes. Variations in intracranial blood volume ${ }^{34}$ may potentially influence also Brain Lymphatic System (BLS) ${ }^{35}$ Recently, Louveau et al. ${ }^{36}$ underlined that the presence of a lymphatic system draining the CNS potentially offers a new player and a new avenue for therapy in neurological disorders, specifically multiple sclerosis and Alzheimer's disease. Similarly Lei Y et al. ${ }^{23}$ emphasized the potential role of ISF to better deliver specific drugs to brain cells taking in account that neural cells occupy only $70-80 \%$ of the total brain volume, the vascular system and ISS together form the brain microenvironment and ISF alone occupies $15-20 \%$ of the total brain volume. Both Authors ${ }^{23-36}$ suggested that neurological afflictions can be viewed as diseases with a neuro-lympho-vascular component and should be therapeutically targeted as such. This kind of approach may be extended to MD treatment, too. The lymphatic vessels run down toward the skull base also along the transverse sinus, the sigmoid sinus, the retrosigmoid vein and the rostral rhinal vein. The endolymphatic 
sac is placed on the posterior surface of the petro us portion of the temporal bone where is in contact with the dura mater. The endolymphatic duct and endolymphatic sac play both an absorptive and secretory role, as well as phagocytic and immunodefensive functions: the endolymphatic sac is capable of antigen recognition and processing for initiation of an immune response. ${ }^{37,38}$ Subject to the above, some brain lymphatic vessels pass through the skull into the nasal mucosa and it is well known the correlation between MD and nose disorders. ${ }^{39}$ Nose and deep cervical lymp nodes therefore are common stations on which converge brain and inner ear waste products both via the GS and the BLS. The case reported in this paper seems strictly connected with the experiments conducted in mice that showed how impairment of these lymph-nodes compromises CNS macromolecule clearance..$^{35}$ The interconnection of brain lymphatics with both the nasal mucosa and the deep cervical lymph-nodes allows T-cells and viruses to easily circulate into the brain $^{40}$ and therefore in the inner ear too. This connection between Brain Lymphatic System and deep cervical limph-nodes seems to support the action of allergic reactions, ${ }^{41}$ viruses and immunological complex ${ }^{42}$ directly arising form the upper airways on the inner ear. Di Berardino et al. ${ }^{43}$ have recently shown that MD patients present also reduced nasal mucous clearance (NCS). ${ }^{44}$ Slowing of clearance of allergens or other potentially dangerous agents from the nose and the upper airways may reduce protective action of cervical lymph-nodes and therefore facilitate sensitization of the inner ear that becomes a hypersensitive target of the immune response to antigen, immuno-complexes and viruses, as speculated it might happen in the CNS. ${ }^{35}$ In other words, tonsillectomy in the reported case, accidentally, produced the removal of specific Infective/irritative foci that sustained MD.

\section{Conclusion}

A Brain Lymphatic System based interpretation of MD pathogenesis leads to a specific therapeutic approach to MD patients because it allows understanding some of the factors to be known playing a significant role in MD.

\section{As a consequence, brain lymphatic system based treatment requires}

i. Prompt and accurate treatment of middle ear infections like otitis media

ii. Accurate treatment of nasal and paranasal sinuses disorders particularly paying attention to restore an effective nasal mucous clearance

iii. Accurate treatment of potentially irritative or infective foci in the head or the neck, e.g tonsillitis or odontostomatological disturbances

iv. Specific allergic desensitization using vaccine for inhalants and appropriate diet for food allergies

v. Prevention of viral re-activation of cranial nerves neuritis, e.g sensor neural sudden hearing loss, vestibular neuritis or facial palsy. ${ }^{45,46}$

\section{Acknowledgements}

None.

\section{Conflict of interest}

Author declares that there is no conflict of interest.

\section{References}

1. James A, Thorp M. Ménière Disease. Clinical Evidence. 2007;3:505.

2. Committee on Hearing and Equilibrium guidelines for the diagnosis and evaluation of therapy in Menière's disease. Otolaryngol Head Neck Surg. 1995;113(3):181-185.

3. Lopez Escamez JA, Carey J, Chung WH, et al. Diagnostic Criteria for Ménière Disease. $J$ Vest Res. 2015;25(1):1-7.

4. Baloh RW. Dizziness, Hearing Loss, and Tinnitus. New York, USA; 1988.

5. Merchant SN, Adams JC, Nadol JB. Patophysiology of Ménière Syndrome: are Symptoms caused by Endolymphatic Hydrops? Otol Neurotol. 2005;26(1):74-81.

6. Schuknecht HF, Ruther A. Blockage of longitudinal flow in endolymphatic hydrops. Eur Arch Otorhinolaryngol. 1991;248(4):209-217.

7. Alpini D, Bavera PM, Hahn A, et al. Chronic Venous cerebrospinal insufficiency (CCSVI) in Meniere Disease. Case or Cause? Science Med. 2013;4:9-12

8. Di Berardino F, Alpini DC, Bavera PM, et al. Chronic cerebrospinal venous insufficiency in Ménière disease. Phlebology. 2015;30(4):274-279.

9. Filipo R Ciciarello F, Attanasio G, Mancini P, et al. chronic cerebrospinal venous insufficiency in patients with Ménière's disease. Eur Arch Otorhinolaryngol. 2015;272(1):77-82.

10. Derebery MJ. Allergic and immunologic aspects of Meniere's disease. Otolaryngol Head Neck Surg. 1996;114(3):360-365.

11. Bergstrom T, Edstrom S, Tjellstrom A, et al. Meniere's disease and antibody reactivity to herpes simplex virus type 1 polypeptides. $\mathrm{Am} \mathrm{J}$ Otolaryngol. 1992;13(5):295-300.

12. Hughes GB, Barna BP, Kinney SE, et al. Autoimmune endolymphatic hydrops: five-year review. Otolaryngol Head Neck Surg. 1988;98(3):221-225.

13. Tomoda K, Suzuka Y, Iwai H, et al. Meniere's disease and autoimmunity: clinical study and survey. Acta Otolaryngologica. 1993;500:31-34.

14. Harcourt JP, Brookes GB. Delayed endolymphatic hydrops: clinical manifestations and treatment outcome. Clin Otolaryngol Allied Sci. 1995;20(4):318-322.

15. Schuknecht HF, Suzuka Y, Zimmermann C. Delayed endolymphatic hydrops and its relationship to Meniere's disease. Ann Otol Rhinol Laryngol. 1990;99(11):843-853.

16. Gacek RR, Gacek MR. Menière's disease as a manifestation of vestibular ganglionitis. Am J Otolaryngol. 2001;22(4):241-250.

17. Gacek RR. Ménière's disease is a viral neuropathy. ORL $J$ Otorhinolaryngol Relat Spec. 2009;71(2):78-86.

18. Di Berardino F, Cesarani A, Hahn A, et al. Viral infection and serum antibodies to heat shock protein 70 in the acute phase of Meniere's disease. Int Tinnitus J. 2007;13(2):90-93.

19. Derebery JM, Berliner KI. Allergy and Its Relation to Menière's Disease. Otolaryngol Clin North Am. 2010;43(5):1047-1058.

20. Savastano M, Giacomelli L, Marioni G. Non-specific immunological determinations in Menière's Disease: any role in clinical practice? Eur Arch Otorhinolaryngol. 2007;264(1):15-19.

21. Di Beradino F, Cesarani A. Gluten Sensitivity in Menière's Disease. Laryngoscope. 2012;122(3):700-702.

22. Di Berardino F, Filipponi E, Alpini D, et al. Ménière disease and gluten sensitivity: recovery after a gluten-free diet. Am J Otolaryngol. 2013;34(4):355-356 
23. Lei Y, Han H, Yuan F, et al. The brain interstitial system:Anatomy, modeling, in vivo measurement, and applications. Prog Neurobiol. 2016;S0301-0082(15):30069-1.

24. Jessen NA, Munk AS, Lundgaard I, et al. The Glymphatic System: A Beginner's Guide. Neurochem Res. 2015;40(12):2583-2599.

25. Wostyn P, Killer HE, De Deyn PP. Glymphatic stasis at the site of the lamina cribrosa as a potential mechanism underlying open-angle glaucoma. Clin Exp Ophthalmol. 2017;45(5):539-547.

26. Alpini DC, Bavera PM, Di Berardino F, et al. Bridging the gap between chronic cerebrospinal venous insufficiency and Ménière Disease. Veins and Lymphatics. 2016:5(2).

27. Xie L, Kang H, Xu Q, et al. Sleep drives metabolite clearance from the adult brain. Science. 2013;342(6156):373-377.

28. Nakayama M, Suzuki M, Inagaki A, et al. Impaired quality of sleep in Meniere's disease patients. J Clin Sleep Med. 2010;6(5):445-449.

29. Platt M, Dilwali S, Elackattu A, et al. Mining immune epitopes in the inner ear. Otolaryngol Head Neck Surg. 2014;150(3):460-463.

30. Louveau A, Smirnov I, Keyes TJ, et al. Structural and functional features of central nervous system lymphatic. Nature. 2015;523(7560):337-341.

31. Goldmann J, Kwidzinski E, Brandt C, et al. T cells traffic from brain to cervical lymph nodes via the cribroid plate and the nasal mucosa. $J$ Leukoc Biol. 2006;80(4):797-801.

32. Aspelund A, Antila S, Proulx ST, et al. A dural lymphatic vascular system that drains brain interstitial fluid and macromolecules. JEM 2015;212(7):991.

33. Raper D, Louveau A, Kipnis J. How Do Meningeal Lymphatic Vessels Drain the CNS? Trends Neurosci. 2016;39(9):581-586.

34. Contarino C, Toro EF. A first step towards a mathematical model for the human Lymphatic System. Paper at 6th ISNVD Annual Meeting, New York, USA; 2016. p. 991-999.
35. Kipnis J. Multifaceted interactions between adaptive immunity and the central nervous system. Science. 2016;353(6301):766-771.

36. Louveau A, Da Mesquita S, Kipnis J. Lymphatics in Neurological Disorders: A Neuro-Lympho-Vascular Component of Multiple Sclerosis and Alzheimer's Disease? Neuron. 2016;91(5):957-973.

37. Moller MN, Kirkeby S, Vikeså J, et al. Gene expression demonstrates an immunological capacity of the human endolymphatic sac. Laryngoscope. 2015;125(8):269-275.

38. Harris JP. Immunology of the inner ear: response of the inner ear to antigen challenge. Otolaryngology--Head and Neck Surgery. 1983;91(1):18-23.

39. Hall CM, Brackman DE. Eustachian tube blockage and Meniere's disease. Arch Otolaryngol. 1977;103(6):355-357.

40. Zamboni P. The discovery of the brain lymphatic system. Veins and Lymphatics. 2015;4(2):5360.

41. Endicott JN, Stucker FJ. Allergy in ménière's disease related fluctuating hearing loss preliminary findings in a double-blind crossover clinical study. Laryngoscope. 1977;87(10):1650-1657.

42. Hsu L, Zhu XN, Zhao YS. Immunoglobulin E and circulating immune complexes in endolymphatic hydrops. Ann Otol Rhinol Laryngol. 1990;99(7):535-538.

43. Di Berardino F, Soi D, Alpini D, et al. Gluten Sensitivity and Meniere Disease. Paper presented at 7 th Symposium on Meniere Disease and Inner Ear Disorders. Italy; 2015.

44. Sleigh MA, Blake JR, Liron N. The propulsion of mucus by cilia. Am Rev Respir Dis. 1988;137(3):726-741.

45. Gacek RR. A perspective on recurrent vertigo. ORL J Otorhinolaryngol Relat Spec. 2013;75(2): 91-107.

46. Gacek RR. Recovery of Hearing in Meniere's Disease after Antiviral Treatment. Am J Otolaryngol. 2015;36(3):315-323. 